\title{
On a New Type of Eisenstein Series in Clifford Analysis
}

\author{
R. S. Kraußhar
}

\begin{abstract}
In this paper we deduce a recursion formula for the partial derivatives of the fundamental solution of the generalized Cauchy-Riemann operator in $\mathbb{R}^{k+1}$ in terms of permutational products. These functions generalize the classical negative power functions to Clifford analysis. We exploit them to introduce a new generalization of the classical complex analytic Eisenstein series on the half-plane to higher dimensions satisfying the generalized Cauchy-Riemann differential equation. Under function-theoretical and number-theoretical aspects we investigate their Fourier series expansion in which multiple divisor sums and certain generalizations of the Riemann zeta function play a crucial role.
\end{abstract}

Keywords: Eisenstein series, Clifford analysis, Riemann zeta function, multiple divisor sums, permutational products

AMS subject classification: $30 \mathrm{G} 35$

\section{Introduction}

In the twentieth century several generalizations of the classical Eisenstein series (cf. [7, 33])

$$
G_{n}(z)=\sum_{(c, d) \in \mathbb{Z} \times \mathbb{Z} \backslash\{(0,0)\}} \frac{1}{(c z+d)^{n}} \quad(n \geq 4, \operatorname{Im}(z)>0)
$$

to higher dimensional spaces have been discussed by several authors related to a rich number of different aspects in function and number theory. C. L. Siegel (cf. [20]) considered generalizations of these series in $\mathbb{C}^{k \times k}$ being endowed with the regularity concept of analyticity of functions in several complex variables. H. Maaß introduced in [26] also non-analytic Eisenstein series in $\mathbb{C}$ by

$$
E_{s}(z)=\frac{1}{2} \sum_{g c d(c, d)=1}\left(\frac{\operatorname{Im}(z)}{\|c z+d\|^{2}}\right)^{s} \quad(s \in \mathbb{C}, \operatorname{Re}(s)>1, \operatorname{Im}(z)>0) .
$$

These non-analytic Eisenstein series are eigenfunctions of the Laplace-Beltrami operator attached to the upper half-plane. Generalizations of them to higher dimensions with respect to certain discrete subgroups of Vahlen's group acting on hyperbolic spaces are

R. S. Kraußhar: Universiteit Gent, Vakgroep Wiskundige Analyse, Galglaan 2, B-9000 Gent krauss@cage.rug.ac.be 
discussed, for example, by J. Elstrodt, F. Mennicke and J. Grunewald (cf. [8, 10]) in the eighties and nineties and by A. Krieg in 1988 in [24, 25]. The generalization presented there, are not hypercomplex analytic in the sense of $[3,6,13,19,30]$. However, they are eigenfunctions of the higher dimensional Laplace-Beltrami operator attached to the upper half-space.

In this paper we deal with a generalization of the classical complex analytic Eisenstein series to hypercomplex analysis which are null-solutions of the generalized CauchyRiemann operator and therefore hypercomplex analytic. The crucial idea is to replace the usual negative power functions by the fundamental solution of the generalized Cauchy-Riemann operator $q_{\mathbf{0}}(z)=\frac{\bar{z}}{\|z\|^{k+1}}$ and by its partial derivatives $q_{\mathbf{n}}(z)=$ $\frac{\partial^{|\mathbf{n}|}}{\partial x^{\mathbf{n}}} q_{\mathbf{0}}(z)$, respectively.

In Section 3 we deduce a recursion formula for the functions $q_{n}$ in terms of permutational products in analogy to H. Malonek's approach for the Fueter polynomials in $[28,29]$. Then, in Section 4, we consider summations of these functions over lattices in $\mathbb{R}^{k+1}$ which lead to generalizations of the Riemann zeta function. With the help of the recursion formula deduced in the previous section, we establish relations to certain Epstein zeta functions. In Section 5 we introduce a generalization of the series $G_{n}$ on a half-space, starting from the Laurent expansion of the generalized monogenic $\wp$-function (see $[6,16-18,22,23,31]$ ) and study basic properties of them. In Section 6 the Fourier expansion of the generalized Eisenstein series is determined explicitly. The first Fourier coefficient turns out to be one of the generalized Riemann zeta functions discussed in Section 4. The other Fourier coefficients are composed by divisor sums. Using multi index notation, the Fourier expansion can be written in a similar form as in the classical complex case. We discuss the influence of monogenicity and compare the monogenic Eisenstein series with the generalizations of Eisenstein series to higher dimensions described in $[8,10,24,25]$.

\section{Preliminaries}

We introduce the most important notions. For detailed information about Clifford algebras and their function theory we refer, for example, to $[1,5,17]$.

By $\left\{e_{1}, e_{2}, \ldots, e_{k}\right\}$ we denote the canonical basis of the Euclidean vector space $\mathbb{R}^{k}$. The attached real Clifford algebra $\mathrm{Cl}_{0 k}$ is the free algebra generated by $\mathbb{R}^{k}$ modulo the relation

$$
\mathbf{x}^{2}=-\|\mathbf{x}\|^{2} e_{0}
$$

where $\mathbf{x} \in \mathbb{R}^{k}$ and $e_{0}$ is the neutral element with respect to multiplication of the Clifford algebra $\mathrm{Cl}_{0 k}$. In the Clifford algebra $\mathrm{Cl}_{0 k}$ the multiplication rules

$$
e_{i} e_{j}+e_{j} e_{i}=-2 \delta_{i j} e_{0} \quad(i, j=1, \ldots, k)
$$

hold where $\delta_{i j}$ is the Kronecker symbol. A basis for the Clifford algebra $\mathrm{Cl}_{0 k}$ is given by the set $\left\{e_{A}: A \subseteq\{1, \ldots, k\}\right\}$ with $e_{A}=e_{l_{1}} e_{l_{2}} \cdots e_{l_{r}}$, where $1 \leq l_{1}<\ldots<l_{r} \leq k$ and $e_{\emptyset}=e_{0}=1$. Every $a \in \mathrm{Cl}_{0 k}$ can be written in the form

$$
a=\sum_{A} a_{A} e_{A} \quad\left(a_{A} \in \mathbb{R}\right) .
$$


Two examples for real Clifford algebras are the complex number field $\mathbb{C}$ and the Hamiltonian skew field $\mathbb{H}$. The conjugation anti-automorphism in the Clifford algebra $\mathrm{Cl}_{0 k}$ is defined by

$$
\bar{a}=\sum_{A} a_{A} \bar{e}_{A}
$$

where $\bar{e}_{A}=\bar{e}_{l_{r}} \bar{e}_{l_{r-1}} \cdots \bar{e}_{l_{1}}$ with $\bar{e}_{j}=-e_{j}$ for $j=1, \ldots, k$ and $\bar{e}_{0}=e_{0}=1$. By

$$
\mathcal{A}_{k+1}=\operatorname{span}_{\mathbb{R}}\left\{1, e_{1}, \ldots, e_{k}\right\}=\mathbb{R} \oplus \mathbb{R}^{k} \subset \mathrm{Cl}_{0 k}
$$

we denote the space of hypercomplex numbers

$$
z=x_{0}+x_{1} e_{1}+x_{2} e_{2}+\cdots+x_{k} e_{k}
$$

often called para-vectors. A para-vector consists only of a scalar part and a vector part.

In this paper we denote pure vectors by a bold face letter, and scalars, para-vectors or Clifford numbers by a normal letter. In this notation the hypercomplex number $z$ is represented in the form $z=x_{0}+\mathbf{x}$ with $\operatorname{Sc}(z)=x_{0}$ and $\operatorname{Vec}(z)=\mathbf{x}$.

We introduce the right half-space of $\mathcal{A}_{k+1}$ as the set of numbers

$$
H^{+}\left(\mathcal{A}_{k+1}\right)=\left\{z \in \mathcal{A}_{k+1} \mid \operatorname{Sc}(z)>0\right\}
$$

and similarly the left half-space. The left and the right half-space are separated by the dividing hyperplane

$$
T=\left\{z \in \mathcal{A}_{k+1} \mid \operatorname{Sc}(z)=0\right\} .
$$

A scalar product between two Clifford numbers $a, b \in \mathrm{Cl}_{0 k}$ is further defined by

$$
\langle a, b\rangle=\operatorname{Sc}(a \bar{b})
$$

and the Clifford norm of an arbitrary $a=\sum_{A} a_{A} e_{A}$ is

$$
\|a\|=\left(\sum_{A}\left|a_{A}\right|^{2}\right)^{\frac{1}{2}} .
$$

Any element $z \in \mathcal{A}_{k+1} \backslash\{0\}$ has an inverse element in $\mathcal{A}_{k+1}$ given by $z^{-1}=\frac{\bar{z}}{\|z\|^{2}}$.

Further, we recall that the permutational product of arbitrary Clifford numbers $a_{1}, \ldots, a_{n}$ is defined by

$$
a_{1} \times a_{2} \times \cdots \times a_{n}=\frac{1}{n !} \sum_{\operatorname{perm}\left(i_{1}, \ldots, i_{n}\right)} a_{i_{1}} \cdot a_{i_{2}} \cdot \cdots \cdot a_{i_{n}} .
$$

For details we refer to $[28,29]$. One further uses the abbreviation

$$
\underbrace{a_{1} \times \cdots \times a_{1}}_{k_{1} \text { times }} \times \cdots \times \underbrace{a_{n} \times \cdots \times a_{n}}_{k_{n} \text { times }}=\left[a_{1}\right]^{k_{1}} \times\left[a_{2}\right]^{k_{2}} \times \cdots \times\left[a_{n}\right]^{k_{n}} .
$$


In order to distinguish powers in terms of the permutational product from powers in the usual sense, one sets brackets when meaning ordinary powers. One has to write for example $\left[a_{1}\right]^{2} \times a_{2}=a_{1} \times a_{1} \times a_{2}$, but $\left(a_{1}\right)^{2} \times a_{2}=\left(a_{1} \cdot a_{1}\right) \times a_{2}$.

In this paper we also deal with complex Clifford algebras. In the same way as a real Clifford algebra is constructed, one can construct a complex Clifford algebra starting from the canonical basis in the complex vector space $\mathbb{C}^{k}$. For details we refer, for example, to [5]. The complex Clifford algebra can be represented by the tensor product $\mathrm{Cl}_{0 k} \otimes_{\mathbb{R}} \mathbb{C}$. Thus, every element $a$ in the complex Clifford algebra $\mathrm{Cl}_{0 k} \otimes_{\mathbb{R}} \mathbb{C}$ can be represented in the form $a=\sum_{A} a_{A} e_{A}$, where $A \subseteq\{1, \ldots, k\}$ and $a_{A} \in \mathbb{C}$. The elements $a_{A} \in \mathbb{C}$ itselves will be written in the form $a_{A}=a_{A_{0}}+i a_{A_{1}}$ with $a_{A_{0}}, a_{A_{1}} \in \mathbb{R}$.

In order to present many calculations in a more suggestive way, the following notations will be used, where $\mathbf{n}=\left(n_{1}, \ldots, n_{k}\right) \in \mathbb{N}_{0}^{k}$ and $\mathbf{j}=\left(j_{1}, \ldots, j_{k}\right) \in \mathbb{N}_{0}^{k}$ are $k$-dimensional multi-indices:

$$
\begin{aligned}
& \mathbf{x}^{\mathbf{n}}=x_{1}^{n_{1}} \cdots x_{k}^{n_{k}}, \quad \mathbf{n} !=n_{1} ! \cdots n_{k} !, \quad|\mathbf{n}|=n_{1}+\ldots+n_{k} \\
& \left(\begin{array}{c}
\mathbf{n} \\
\mathbf{j}
\end{array}\right)=\left(\begin{array}{c}
n_{1} \\
j_{1}
\end{array}\right) \cdots\left(\begin{array}{c}
n_{k} \\
j_{k}
\end{array}\right) \\
& \mathbf{j} \leq \mathbf{n} \Leftrightarrow \quad j_{1} \leq n_{1}, \ldots, j_{k} \leq n_{k} .
\end{aligned}
$$

By $\tau(i)$ we denote the multi-index $\left(n_{1}, \ldots, n_{k}\right)$ with $n_{j}=\delta_{i j}$ for $1 \leq j \leq k$.

A. C. Dixon [6], R. Fueter [17], G. C. Moisil and N. Theodorescu [30], V. Iftimie [19] and R. Delanghe [3] are some of the most important creators of a function theory in Clifford algebras. In $\mathcal{A}_{k+1}$ one considers the generalized Cauchy-Riemann operator

$$
D=\frac{\partial}{\partial x_{0}}+\sum_{i=1}^{k} \frac{\partial}{\partial x_{i}} e_{i}
$$

Suppose $U \subset \mathcal{A}_{k+1}$ is open. Then a real differentiable function $f: U \rightarrow \mathrm{Cl}_{0 k}$ is called left (right) monogenic at a point $z_{0} \in U$ if $D f\left(z_{0}\right)=0$ or $f D\left(z_{0}\right)=0$, respectively. The notion of left (right) monogenicity in $\mathcal{A}_{k+1}$ provides a generalization of the concept of complex analyticity to Clifford analysis in the sense of the Cauchy-Riemann approach. This concept is often called hypercomplex analyticity.

Many classical theorems from complex analysis could be generalized to higher dimensions by this approach. We refer, e.g., to [1, 17]. However, because of the noncommutativity in Clifford algebras, the positive and negative powers of the hypercomplex variable $z$ are not monogenic. In hypercomplex function theory the positive powers are substituted by the following polynomials, mentioned first in [14] and therefore often called Fueter polynomials:

$$
V_{\mathbf{n}}(z)=\frac{1}{|\mathbf{n}| !} \sum_{\pi \in \operatorname{perm}(\mathbf{n})} z_{\pi\left(n_{1}\right)} z_{\pi\left(n_{2}\right)} \cdots z_{\pi\left(n_{k}\right)}
$$

where $\operatorname{perm}(\mathbf{n})$ denotes the set of all permutations of the sequence $\left(n_{1}, \ldots, n_{k}\right)$ and $z_{i}=x_{i}-x_{0} e_{i}$ for $i=1, \ldots, k$ and $V_{\mathbf{0}}(z)=1$. H. Malonek has shown in $[28,29]$ that one 
can write the Fueter polynomials in terms of permutational products of the variables $z_{i}$, more precisely

$$
V_{n_{1}, \ldots, n_{k}}(z)=\frac{1}{\mathbf{n} !}\left[z_{1}\right]^{n_{1}} \times \cdots \times\left[z_{k}\right]^{n_{k}} .
$$

The negative powers are substituted (cf. [4, 14]) by the function

$$
q_{\mathbf{0}}(z)=\frac{\bar{z}}{\|z\|^{k+1}} \quad \text { and } \quad q_{\mathbf{n}}(z)=\frac{\partial^{|\mathbf{n}|}}{\partial \mathbf{x}^{\mathbf{n}}} q_{\mathbf{0}}(z) \quad(|\mathbf{n}| \geq 1)
$$

In the next section we will show that we can also write the functions $q_{\mathbf{n}}$ in terms of permutational products.

It is important to mention that the set of left (right) monogenic functions forms just a Clifford right (left) module for $k>1$. The product, the quotient or the composition of two monogenic functions gives in general no monogenic function for $k>1$. However, supposed that $f$ is a left (right) monogenic function and if further $\omega \in \mathcal{A}_{k+1}$ and $c \in \mathbb{R} \backslash\{0\}$, then $F(z)=f(c z+\omega)$ is a left (right) monogenic function in the variable $z$.

\section{A recursion formula for the $q_{n}$-functions}

In this section we deduce a recursion formula for the functions $q_{\mathbf{n}}$ in terms of permutational products. The representation of the $q_{\mathbf{n}}$-functions that we will obtain in this section provides an analogy to H. Malonek's representation of the Fueter polynomials $V_{\mathbf{n}}$ in $[28,29]$.

We first prove

Lemma 1. Suppose $n \in \mathbb{N}$. Then

$$
\begin{aligned}
\frac{\partial^{n}}{\partial x_{1}^{n}} q_{\mathbf{0}}(z)= & \sum_{j=0}^{n-1}\left(\begin{array}{c}
n-1 \\
j
\end{array}\right) j ! q_{(n-(j+1)) \tau(1)}(z) \\
& \cdot\left[\left(\frac{k-1}{2}\right)\left(\bar{z}^{-1} e_{1}\right)^{j+1}+(-1)^{j+1}\left(\frac{k+1}{2}\right)\left(e_{1} z^{-1}\right)^{j+1}\right]
\end{aligned}
$$

Proof. We prove this lemma by induction. By a direct computation, we obtain

$$
\begin{aligned}
q_{\tau(1)}(z) & =-\frac{e_{1}}{\|z\|^{k+1}}+\frac{k+1}{2} \frac{e_{1}}{\|z\|^{k+1}}-\frac{k+1}{2} \frac{\bar{z} e_{1} \bar{z}}{\|z\|^{k+3}} \\
& =q_{\mathbf{0}}(z)\left[\frac{k-1}{2} \bar{z}^{-1} e_{1}-\frac{k+1}{2} e_{1} z^{-1}\right]
\end{aligned}
$$

Thus, the assertion is true for $n=1$.

In the sequel we assume $n \geq 1$. For $n \in \mathbb{N}$ we get by induction

$$
\frac{\partial^{n}}{\partial x_{1}^{n}}\left\{\bar{z}^{-1}\right\}=n !\left(\bar{z}^{-1} e_{1}\right)^{n} \bar{z}^{-1} \quad \text { and } \quad \frac{\partial^{n}}{\partial x_{1}^{n}}\left\{z^{-1}\right\}=(-1)^{n} n ! z^{-1}\left(e_{1} z^{-1}\right)^{n} \text {. }
$$


Hence, we obtain

$$
\begin{aligned}
& q_{(n+1) \tau(1)}(z)=\frac{\partial}{\partial x_{1}} \sum_{j=0}^{n-1}\left(\begin{array}{c}
n-1 \\
j
\end{array}\right) j ! q_{(n-(j+1)) \tau(1)}(z) \\
& \cdot\left[\left(\frac{k-1}{2}\right)\left(\bar{z}^{-1} e_{1}\right)^{j+1}+(-1)^{j+1}\left(\frac{k+1}{2}\right)\left(e_{1} z^{-1}\right)^{j+1}\right] \\
& =\sum_{j=0}^{n-1}\left(\begin{array}{c}
n-1 \\
j
\end{array}\right) j !\left\{q_{(n-j) \tau(1)}(z)\right. \\
& \cdot\left[\left(\frac{k-1}{2}\right)\left(\bar{z}^{-1} e_{1}\right)^{j+1}+(-1)^{j+1}\left(\frac{k+1}{2}\right)\left(e_{1} z^{-1}\right)^{j+1}\right] \\
& +q_{(n-(j+1)) \tau(1)}(z) \\
& \text {. } \left.\left[\left(\frac{k-1}{2}\right)(j+1)\left(\bar{z}^{-1} e_{1}\right)^{j+2}+(-1)^{j+2}\left(\frac{k+1}{2}\right)(j+1)\left(e_{1} z^{-1}\right)^{j+2}\right]\right\} \\
& =\sum_{j=0}^{n-1}\left(\begin{array}{c}
n-1 \\
j
\end{array}\right) j ! q_{(n-j) \tau(1)}(z) \\
& \cdot\left[\left(\frac{k-1}{2}\right)\left(\bar{z}^{-1} e_{1}\right)^{j+1}+(-1)^{j+1}\left(\frac{k+1}{2}\right)\left(e_{1} z^{-1}\right)^{j+1}\right] \\
& +\sum_{j=1}^{n}\left(\begin{array}{c}
n-1 \\
j-1
\end{array}\right) j ! q_{(n-j) \tau(1)}(z) \\
& \cdot\left[\left(\frac{k-1}{2}\right)\left(\bar{z}^{-1} e_{1}\right)^{j+1}+(-1)^{j+1}\left(\frac{k+1}{2}\right)\left(e_{1} z^{-1}\right)^{j+1}\right] \\
& =\sum_{j=0}^{n}\left(\begin{array}{c}
n \\
j
\end{array}\right) j ! q_{(n-j) \tau(1)}(z) \\
& \cdot\left[\left(\frac{k-1}{2}\right)\left(\bar{z}^{-1} e_{1}\right)^{j+1}+(-1)^{j+1}\left(\frac{k+1}{2}\right)\left(e_{1} z^{-1}\right)^{j+1}\right]
\end{aligned}
$$

and the proof is finished

Analogously we may proceed for the determination of

$$
\frac{\partial^{n_{i}}}{\partial x_{i}^{n_{i}}} q_{\mathbf{0}}
$$

for $i=2, \ldots, k$. We get the same recursion formula replacing simply $e_{1}$ by $e_{i}$.

Now we want to establish a recursion formula, where $\mathbf{n}$ is a general multi-index of $\mathbb{N}_{0}^{k} \backslash\{\mathbf{0}\}$. As soon as we deal with mixed derivatives, permutational products appear. We will illustrate this by the following examples.

We assume $i, j \in\{1, \ldots, k\}$ to be distinct. Then we get

$$
\begin{aligned}
\frac{\partial}{\partial x_{j}} \frac{\partial}{\partial x_{i}} q_{\mathbf{0}}(z)= & q_{\tau(j)}(z)\left[\left(\frac{k-1}{2}\right)\left(\bar{z}^{-1} e_{i}\right)-\left(\frac{k+1}{2}\right)\left(e_{i} z^{-1}\right)\right] \\
& +q_{\mathbf{0}}(z)\left[\left(\frac{k-1}{2}\right)\left(\bar{z}^{-1} e_{j}\right)\left(\bar{z}^{-1} e_{i}\right)+\left(\frac{k+1}{2}\right)\left(e_{i} z^{-1}\right)\left(e_{j} z^{-1}\right)\right]
\end{aligned}
$$


and further

$$
\begin{aligned}
\frac{\partial^{3} q_{\mathbf{0}}(z)}{\partial x_{j} \partial x_{i}^{2}}= & q_{\tau(j)+\tau(i)}(z)\left[\left(\frac{k-1}{2}\right)\left(\bar{z}^{-1} e_{i}\right)-\left(\frac{k+1}{2}\right)\left(e_{i} z^{-1}\right)\right] \\
& +q_{\tau(i)}(z)\left[\left(\frac{k-1}{2}\right)\left(\bar{z}^{-1} e_{j}\right)\left(\bar{z}^{-1} e_{i}\right)+\left(\frac{k+1}{2}\right)\left(e_{i} z^{-1}\right)\left(e_{j} z^{-1}\right)\right] \\
& +q_{\tau(j)}(z)\left[\left(\frac{k-1}{2}\right)\left(\bar{z}^{-1} e_{i}\right)^{2}+\left(\frac{k+1}{2}\right)\left(e_{i} z^{-1}\right)^{2}\right] \\
& +2 q_{\mathbf{0}}(z)\left[\left(\frac{k-1}{2}\right)\left[\bar{z}^{-1} e_{j}\right] \times\left[\bar{z}^{-1} e_{i}\right] \cdot\left(\bar{z}^{-1} e_{i}\right)\right. \\
& \left.-\left(\frac{k+1}{2}\right)\left(e_{i} z^{-1}\right) \cdot\left[e_{i} z^{-1}\right] \times\left[e_{j} z^{-1}\right]\right]
\end{aligned}
$$

For the iteration of this procedure we deduce by a simple induction proof the following formulas:

$$
\begin{aligned}
& \frac{\partial}{\partial x_{j}}\left\{\left[\bar{z}^{-1} e_{i}\right]^{n_{i}} \times\left[\bar{z}^{-1} e_{j}\right]^{n_{j}} \cdot\left(\bar{z}^{-1} e_{i}\right)\right\} \\
& =\left(n_{i}+n_{j}+1\right)\left[\bar{z}^{-1} e_{i}\right]^{n_{i}} \times\left[\bar{z}^{-1} e_{j}\right]^{n_{j}+1} \cdot\left(\bar{z}^{-1} e_{i}\right) \\
& \frac{\partial}{\partial x_{j}}\left\{\left(e_{i} z^{-1}\right) \cdot\left[e_{i} z^{-1}\right]^{n_{i}} \times\left[z^{-1} e_{j}\right]^{n_{j}}\right\} \\
& =-\left(n_{i}+n_{j}+1\right)\left(e_{i} z^{-1}\right) \cdot\left[e_{i} z^{-1}\right]^{n_{i}} \times\left[e_{j} z^{-1}\right]^{n_{j}+1} .
\end{aligned}
$$

With these formulas we can show finally the following theorem using the notation

$$
\sum_{\mathbf{0} \leq \mathbf{j} \leq \mathbf{n}}=\sum_{j_{1}=0}^{n_{1}} \sum_{j_{2}=0}^{n_{2}} \cdots \sum_{j_{k}=0}^{n_{k}} .
$$

Theorem 1 (Representation of the $q_{\mathbf{n}}$-functions in terms of permutational products). Let $\mathbf{n}=\left(n_{1}, n_{2}, n_{3}, \ldots, n_{k}\right) \in \mathbb{N}_{0}^{k}$. Then

$$
\begin{aligned}
q_{\mathbf{n}+\tau(1)}(z)= & \sum_{\mathbf{0} \leq \mathbf{j} \leq \mathbf{n}}\left(\begin{array}{c}
\mathbf{n} \\
\mathbf{j}
\end{array}\right)|\mathbf{j}| ! q_{\mathbf{n}-\mathbf{j}}(z) \\
& \cdot\left[\left(\frac{k-1}{2}\right)\left[\bar{z}^{-1} e_{k}\right]^{j_{k}} \times \cdots \times\left[\bar{z}^{-1} e_{1}\right]^{j_{1}} \cdot\left(\bar{z}^{-1} e_{1}\right)\right. \\
& \left.+(-1)^{|\mathbf{j}|+1}\left(\frac{k+1}{2}\right)\left(e_{1} z^{-1}\right) \cdot\left[e_{1} z^{-1}\right]^{j_{1}} \times \cdots \times\left[e_{k} z^{-1}\right]^{j_{k}}\right] .
\end{aligned}
$$

Proof. For multi-indices of the form $\mathbf{n}=\left(n_{1}, 0, \ldots, 0\right)$ with $n_{1} \in \mathbb{N}_{0}$, the statement is true according to Lemma 1. Suppose now $\mathbf{n}$ to be a multi-index of the form $\mathbf{n}=$ $\left(n_{1}, n_{2}, 0, \ldots, 0\right)$ where $n_{2} \in \mathbb{N}_{0}$. For $n_{2}=0$ the statement is true according to Lemma 1. By a direct computation one verifies immediately that the assertion holds also for 
$n_{2}=1$. In the sequel we assume $n_{2} \geq 1$ and compute

$$
\begin{aligned}
& \frac{\partial}{\partial x_{2}} q_{n_{1}+1, n_{2}, 0, \ldots, 0}(z) \\
& =\sum_{\mathbf{0} \leq \mathbf{j} \leq \mathbf{n}}\left(\begin{array}{l}
\mathbf{n} \\
\mathbf{j}
\end{array}\right)|\mathbf{j}| ! q_{n_{1}-j_{1}, n_{2}+1-j_{2}, 0, \ldots, 0}(z) \\
& \cdot\left[\left(\frac{k-1}{2}\right)\left[\bar{z}^{-1} e_{2}\right]^{j_{2}} \times\left[\bar{z}^{-1} e_{1}\right]^{j_{1}} \cdot\left(\bar{z}^{-1} e_{1}\right)\right. \\
& \left.+(-1)^{|\mathbf{j}|+1}\left(\frac{k+1}{2}\right)\left(e_{1} z^{-1}\right) \cdot\left[e_{1} z^{-1}\right]^{j_{1}} \times\left[e_{2} z^{-1}\right]^{j_{2}}\right] \\
& +\sum_{\mathbf{0} \leq \mathbf{j} \leq \mathbf{n}}\left(\begin{array}{c}
\mathbf{n} \\
\mathbf{j}
\end{array}\right)|\mathbf{j}| ! q_{n_{1}-j_{1}, n_{2}-j_{2}, 0, \ldots, 0}(z) \\
& \cdot(|\mathbf{j}|+1)\left[\left(\frac{k-1}{2}\right)\left[\bar{z}^{-1} e_{2}\right]^{j_{2}+1} \times\left[\bar{z}^{-1} e_{1}\right]^{j_{1}} \cdot\left(\bar{z}^{-1} e_{1}\right)\right. \\
& \left.+(-1)^{|\mathbf{j}|+2}\left(\frac{k+1}{2}\right)\left(e_{1} z^{-1}\right) \cdot\left[e_{1} z^{-1}\right]^{j_{1}} \times\left[e_{2} z^{-1}\right]^{j_{2}+1}\right] \\
& =\sum_{\mathbf{0} \leq \mathbf{j} \leq \mathbf{n}}\left(\begin{array}{c}
\mathbf{n} \\
\mathbf{j}
\end{array}\right)|\mathbf{j}| ! q_{n_{1}-j_{1},\left(n_{2}+1\right)-j_{2}, 0, \ldots, 0}(z) \\
& \cdot\left[\left(\frac{k-1}{2}\right)\left[\bar{z}^{-1} e_{2}\right]^{j_{2}} \times\left[\bar{z}^{-1} e_{1}\right]^{j_{1}} \cdot\left(\bar{z}^{-1} e_{1}\right)\right. \\
& \left.+(-1)^{|\mathbf{j}|+1}\left(\frac{k+1}{2}\right)\left(e_{1} z^{-1}\right) \cdot\left[e_{1} z^{-1}\right]^{j_{1}} \times\left[e_{2} z^{-1}\right]^{j_{2}}\right] \\
& \stackrel{j_{2} \leftarrow j_{2}+1}{+} \sum_{\tau(2) \leq \mathbf{j} \leq \mathbf{n}+\tau(2)}\left(\begin{array}{c}
\mathbf{n} \\
\mathbf{j}-\tau(2)
\end{array}\right)|\mathbf{j}| ! q_{n_{1}-j_{1}, n_{2}+1-j_{2}, 0 \ldots, 0}(z) \\
& \cdot\left[\left(\frac{k-1}{2}\right)\left[\bar{z}^{-1} e_{2}\right]^{j_{2}} \times\left[\bar{z}^{-1} e_{1}\right]^{j_{1}} \cdot\left(\bar{z}^{-1} e_{1}\right)\right. \\
& \left.+(-1)^{|\mathbf{j}|+1}\left(\frac{k+1}{2}\right)\left(e_{1} z^{-1}\right) \cdot\left[e_{1} z^{-1}\right]^{j_{1}} \times\left[e_{2} z^{-1}\right]^{j_{2}}\right] \\
& =\sum_{\mathbf{0} \leq \mathbf{j} \leq \mathbf{n}+\tau(2)}\left(\begin{array}{c}
\mathbf{n}+\tau(2) \\
\mathbf{j}
\end{array}\right)|\mathbf{j}| ! q_{n_{1}-j_{1},\left(n_{2}+1\right)-j_{2}, 0, \ldots, 0}(z) \\
& \cdot\left[\left(\frac{k-1}{2}\right)\left[\bar{z}^{-1} e_{2}\right]^{j_{2}} \times\left[\bar{z}^{-1} e_{1}\right]^{j_{1}} \cdot\left(\bar{z}^{-1} e_{1}\right)\right. \\
& \left.+(-1)^{|\mathbf{j}|+1}\left(\frac{k+1}{2}\right)\left(e_{1} z^{-1}\right) \cdot\left[e_{1} z^{-1}\right]^{j_{1}} \times\left[e_{2} z^{-1}\right]^{j_{2}}\right] .
\end{aligned}
$$

So, the assertion is also true for $n_{2}+1$. Thus, the formula is proved for all indices $\mathbf{n}$ of the form $\left(n_{1}, n_{2}, 0, \ldots, 0\right)$ with $\left(n_{1}, n_{2}\right) \in \mathbb{N}_{0}^{2}$.

Next we assume $\mathbf{n}$ to have the form $\mathbf{n}=\left(n_{1}, n_{2}, n_{3}, 0, \ldots, 0\right)$ with $\left(n_{1}, n_{2}, n_{3}\right) \in \mathbb{N}_{0}^{3}$. For multi-indices $\mathbf{n}=\left(n_{1}, n_{2}, n_{3}, 0, \ldots, 0\right)$ with $n_{3}=0$ the validity of the formula has been shown by the previous induction step. With induction over $n_{3}$ one verifies 
analogously to the previous induction procedure that the formula holds for all multiindices of the form $\left(n_{1}, n_{2}, n_{3}, 0, \cdots, 0\right)$. Successively one proceeds to consider then multi-indices $\mathbf{n}$ of the form $\left(n_{1}, n_{2}, n_{3}, n_{4}, 0, \ldots, 0\right)$, proves the formula by induction over $n_{4}$ analogously as we have shown in the second step, and proceeds with further induction steps until one finally obtains the validity of the formula for all multi-indices $\left(n_{1}, n_{2}, \ldots, n_{k}\right) \in \mathbb{N}_{0}^{k}$

For the sake of completeness, it remains to derive a formula for the functions $q_{\mathbf{m}}$ where $\mathbf{m}:=\left(m_{1}, \ldots, m_{k}\right)$ is a multi-index with $m_{1}=0$. In this case one chooses an $\alpha \in\{2, \ldots, k\}$ with $m_{\alpha} \neq 0$. In the associated formula for the function $q_{\mathbf{m}}$ the index $\alpha$ plays then the role of the index 1 in Theorem 1 . The following corollary provides a more precise formulation and furthermore a correction of [22: Lemma 1.2]:

Corollary 1. Let $\alpha \in\{1, \ldots, k\}$ and $\mathbf{n} \in \mathbb{N}_{0}^{k}$. Then

$$
\begin{aligned}
q_{\mathbf{n}+\tau(\alpha)}= & \sum_{\mathbf{0} \leq \mathbf{j} \leq \mathbf{n}}\left(\begin{array}{c}
\mathbf{n} \\
\mathbf{j}
\end{array}\right)|\mathbf{j}| ! q_{\mathbf{n}-\mathbf{j}}(z) \\
& \cdot\left[\left(\frac{k-1}{2}\right)\left[\bar{z}^{-1} e_{k}\right]^{j_{k}} \times \cdots \times\left[\bar{z}^{-1} e_{1}\right]^{j_{1}} \cdot\left(\bar{z}^{-1} e_{\alpha}\right)\right. \\
& \left.+(-1)^{|\mathbf{j}|+1}\left(\frac{k+1}{2}\right)\left(e_{\alpha} z^{-1}\right) \cdot\left[e_{1} z^{-1}\right]^{j_{1}} \times \cdots \times\left[e_{k} z^{-1}\right]^{j_{k}}\right] .
\end{aligned}
$$

As a first application of this recursion formula we get the following estimates on $q_{\mathbf{n}}$-functions.

Proposition 1. For all multi-indices $\mathbf{n} \in \mathbb{N}_{0}^{k}$ the estimate

$$
\left\|\frac{\partial^{|\mathbf{n}|}}{\partial \mathbf{x}^{\mathbf{n}}} q_{\mathbf{0}}(z)\right\| \leq \frac{k(k+1) \cdots(k+|\mathbf{n}|-1)}{\|z\|^{k+|\mathbf{n}|}}
$$

holds for all $z \in \mathcal{A}_{k+1}$.

Proof. We first restrict ourselfes to the case where $\mathbf{n}=(n, 0, \ldots, 0)$. A simple calculation shows that the estimate holds for $n=0$ and $n=1$. In the sequel we assume $n \geq 1$ and consider

$$
\begin{aligned}
\left\|\frac{\partial^{n+1}}{\partial x_{1}^{n+1}} q_{\mathbf{0}}(z)\right\|= & \| \sum_{j=0}^{n}\left(\begin{array}{c}
n \\
j
\end{array}\right) j ! q_{(n-j) \tau(1)}(z) \\
& \cdot\left[\left(\frac{k-1}{2}\right)\left(\bar{z}^{-1} e_{1}\right)^{j+1}+(-1)^{j+1}\left(\frac{k+1}{2}\right)\left(e_{1} z^{-1}\right)^{j+1}\right] \| \\
\leq & \frac{k}{\|z\|^{k+n+1}} n !\left[\sum_{j=0}^{n} \frac{((k-1)+(n-j)) !}{(k-1) !(n-j) !}\right] \\
= & \frac{k}{\|z\|^{k+n+1}} n !\left[\sum_{j=0}^{n}\left(\begin{array}{c}
(k-1)+n-j \\
n-j
\end{array}\right)\right]
\end{aligned}
$$




$$
\begin{aligned}
& =\frac{k}{\|z\|^{k+n+1}} n !\left(\begin{array}{c}
k+n \\
n
\end{array}\right) \\
& =\frac{k}{\|z\|^{k+n+1}} \frac{(k+n) !}{k !} \\
& =k(k+1) \cdots(k+n) \frac{1}{\|z\|^{k+n+1}} .
\end{aligned}
$$

Thus, the assertion holds for indices of the form $\mathbf{n}=(n, 0, \ldots, 0)$.

With the estimates

$$
\begin{aligned}
& \left\|\left(e_{1} z^{-1}\right) \cdot\left[e_{1} z^{-1}\right]^{j_{1}} \times \cdots \times\left[e_{k} z^{-1}\right]^{j_{k}}\right\| \leq\left\|e_{1} z^{-1}\right\||\mathbf{j}|+1 \\
& \left\|\left[\bar{z}^{-1} e_{k}\right]^{j_{k}} \times \cdots \times\left[\bar{z}^{-1} e_{1}\right]^{j_{1}} \cdot\left(\bar{z}^{-1} e_{1}\right)\right\| \leq\left\|\bar{z}^{-1} e_{1}\right\|^{|\mathbf{j}|+1}
\end{aligned}
$$

and the formula

$$
\sum_{\substack{\mathbf{j} \in \mathbb{N}_{0}^{k} \\
|\mathbf{j}|=j}}\left(\begin{array}{c}
n_{1} \\
j_{1}
\end{array}\right) \cdots\left(\begin{array}{c}
n_{k} \\
j_{k}
\end{array}\right)=\left(\begin{array}{c}
|\mathbf{n}| \\
j
\end{array}\right)
$$

in combination with the statement of Theorem 1 or Corollary 1, respectively, we get further, applying a simple induction argument, that

$$
\left\|q_{\mathbf{n}}(z)\right\| \leq\left\|q_{|\mathbf{n}|, 0, \cdots, 0}(z)\right\| \quad\left(\mathbf{n} \in \mathbb{N}_{0}^{k}\right)
$$

and the assertion is shown

\section{Remarks.}

1. Since the functions $q_{\mathbf{n}}$ are $\mathbb{R}$-homogeneous polynomials of degree $-(k+|\mathbf{n}|)$ (see [1]) one obtains directly an inequality of the general type

$$
\left\|q_{\mathbf{n}}(z)\right\| \leq C(\mathbf{n})\|z\|^{-(k+|\mathbf{n}|)}
$$

with a constant $C(\mathbf{n}) \in \mathbb{R}^{>0}$ being dependent on $\mathbf{n}$. As a direct consequence one gets

$$
\lim _{z \rightarrow \infty} q_{\mathbf{n}}(z)=0
$$

2. In $[15,17]$ R. Fueter proved for the quaternionic case that

$$
\left\|q_{\mathbf{n}}(z)\right\| \leq(|\mathbf{n}|+2) !\|z\|^{-(|\mathbf{n}|+3)}
$$

He used a different method to obtain this inequality. His proof is based on the formula

$$
q_{\mathbf{n}}(\zeta)=\zeta^{-1} \frac{\partial^{|\mathbf{n}|}}{\partial \mathbf{x}^{\mathbf{n}}}\left[\Delta_{z}\left\{\left(z \zeta^{-1}\right)^{n+2}\right\}\right] \quad(z \in \mathbb{H}, \zeta \in \mathbb{H} \backslash\{0\})
$$

where $\Delta_{z}$ denotes the Laplace operator with respect to the variable $z$. We observe that the estimate in Proposition 1 for the quaternionic case is stronger than R. Fueter's estimate. 


\section{The generalized Riemann zeta function associated with the $q_{\mathbf{n}}$-functions}

Now we proceed in the direction to consider summations of the functions $q_{\mathbf{n}}(\omega)$ over a lattice in $\mathcal{A}_{k+1}$ which leads to a generalization of the Riemann zeta function.

The general form of a $p$-dimensional lattice in $\mathcal{A}_{k+1}$ can be presented by

$$
\Omega_{p}=\mathbb{Z} \omega_{1}+\ldots+\mathbb{Z} \omega_{p} \quad(1 \leq p \leq k+1)
$$

where $\omega_{1}, \ldots, \omega_{p} \in \mathcal{A}_{k+1}$ are supposed to be $\mathbb{R}$-linear independent para-vectors. For the following applications we need G. Eisenstein's lemma formulated in the para-vector formalism:

Lemma 2 (cf. $[7,31]$ ). Suppose $a_{0}, a_{1}, \ldots, a_{t}$ are $\mathbb{R}$-linear independent para-vectors in $\mathcal{A}_{k+1}$. Then the series

$$
\sum_{\left(m_{0}, m_{1}, \ldots, m_{t}\right) \in \mathbb{Z}^{t+1} \backslash\{\mathbf{0}\}}\left\|m_{0} a_{0}+m_{1} a_{1}+\ldots+m_{t} a_{t}\right\|^{-(t+\alpha)}
$$

converges if and only if $\alpha \geq 2$.

We further split the lattice into a positive and negative part.

Definition 1. To a given $p$-dimensional lattice (6) in $\mathcal{A}_{k+1}$, the positive semi-lattice $\Omega_{p}^{+}$is defined by

$$
\begin{array}{rrr}
\Omega_{p}^{+}= & \mathbb{N} \omega_{1}+\mathbb{Z} \omega_{2}+\mathbb{Z} \omega_{3}+\ldots+\mathbb{Z} \omega_{p} \\
& \cup & \mathbb{N} \omega_{2}+\mathbb{Z} \omega_{3}+\ldots+\mathbb{Z} \omega_{p} \\
& \vdots & \\
& \cup & \mathbb{N} \omega_{p} .
\end{array}
$$

The negative part $\Omega_{p}^{-}$of the lattice $\Omega_{p}$ is defined by

$$
\Omega_{p}^{-}=\left(\Omega_{p} \backslash\{0\}\right) \backslash \Omega_{p}^{+} .
$$

Remarks. We observe that

$$
z \in \Omega_{p}^{+} \Leftrightarrow-z \in \Omega_{p}^{-} \quad \text { and } \quad \Omega_{p}^{+} \cup \Omega_{p}^{-} \cup\{0\}=\Omega_{p} \text {. }
$$

In particular, for $k=1$ and $\omega_{1}=1$ one gets

$$
\Omega_{1}^{+}=\mathbb{N}, \quad \Omega_{1}^{-}=-\mathbb{N}, \quad \Omega_{1}=\mathbb{N} \cup-\mathbb{N} \cup\{0\}=\mathbb{Z} .
$$

With this notation we introduce 
Definition 2 (Generalized Riemann zeta function of Clifford analysis in $\mathcal{A}_{k+1}$ ). Let $p \in \mathbb{N}$ with $1 \leq p \leq k+1$. Let further $\mathbf{l} \in \mathbb{N}_{0}^{k}$ be a multi-index and suppose for $p=k$ that $|\mathbf{l}| \geq 1$ and for $p=k+1$ that $|\mathbf{l}| \geq 2$. Then the generalized Riemann zeta function of Clifford analysis in $\mathcal{A}_{k+1}$ is defined by

$$
\zeta_{M}^{\Omega_{p}}(\mathbf{l})=\sum_{\omega \in \Omega_{p}^{+}} q_{\mathbf{l}}(\omega) .
$$

The series converges absolutely which follows by Lemma 2 after having applied Proposition 1. Note that in the case $|\mathbf{l}| \equiv 1(2)$ we obtain

$$
2 \zeta_{M}^{\Omega_{p}}(\mathbf{l})=\sum_{\omega \in \Omega_{p} \backslash\{0\}} q_{\mathbf{l}}(\omega) .
$$

Now we want to discuss a simple example of the generalized Riemann zeta function which illustrates that these functions are closely related to Epstein zeta functions. For convenience we recall (cf. [21]) that the Epstein zeta function associated with a given $(p \times p)$ positive definite symmetric matrix $S$ is said to be

$$
\zeta_{S}(s)=\sum_{\mathbf{g} \in \mathbb{Z}^{p} \backslash\{\mathbf{0}\}}\left(\mathbf{g}^{t r} S \mathbf{g}\right)^{-s} \quad\left(s \in \mathbb{C}, \operatorname{Re}(s)>\frac{p}{2}\right) .
$$

In the sequel we consider a $p$-dimensional lattice with $p \leq k$ and $S c\left(\Omega_{p}\right)=0$. The simplest non-trivial example for the generalized Riemann zeta function related to this lattice is

$$
\zeta_{M}^{\Omega_{p}}(\tau(i))=\frac{1}{2} \sum_{\omega \in \Omega_{p} \backslash\{0\}} q_{\tau(i)}(\omega) .
$$

According to Lemma 1, we know

$$
q_{\tau(i)}(z)=q_{\mathbf{0}}(z)\left[\left(\frac{k-1}{2}\right)\left(\bar{z}^{-1} e_{i}\right)-\left(\frac{k+1}{2}\right)\left(e_{i} z^{-1}\right)\right] .
$$

Therefore, we obtain the representation

$$
\begin{aligned}
\zeta_{M}^{\Omega_{p}}(\tau(i)) & =\frac{1}{2} \sum_{\omega \in \Omega_{p} \backslash\{0\}} \frac{\omega}{\|\omega\|^{k+1}}\left[\left(\frac{k-1}{2}\right) \omega^{-1} e_{i}+\left(\frac{k+1}{2}\right) e_{i} \omega^{-1}\right] \\
& =\frac{1}{4} \sum_{\omega \in \Omega_{p} \backslash\{0\}}\left[\frac{(k-1) e_{i}}{\|\omega\|^{k+1}}+\frac{(k+1) \omega e_{i} \bar{\omega}}{\|\omega\|^{k+3}}\right] \\
& =\frac{k-1}{4} e_{i} \zeta_{W^{t r} W}\left(\frac{1}{2}(k+1)\right)+\frac{k+1}{4} \sum_{\omega \in \Omega_{p} \backslash\{0\}} \frac{\omega e_{i} \bar{\omega}}{\|\omega\|^{k+3}}
\end{aligned}
$$

where $W=\left(w_{1}, \ldots, w_{p}\right)$ since the series

$$
\sum_{\omega \in \Omega_{p} \backslash\{0\}} \frac{1}{\|\omega\|^{k+1}} \quad \text { and } \quad \sum_{\omega \in \Omega_{p} \backslash\{0\}} \frac{\omega e_{i} \bar{\omega}}{\|\omega\|^{k+3}}
$$


converge both absolutely.

In particular, one obtains for the quaternionic case

$$
\zeta_{M}^{\Omega_{p}}(\tau(l))=\frac{1}{2} i_{l} \zeta_{W^{t r} W}(2)+\sum_{\omega \in \Omega_{p} \backslash\{0\}} \frac{\omega i_{l} \bar{\omega}}{\|\omega\|^{6}}
$$

where $i_{l}$ denotes the $l$-th imaginary unit in the quaternionic skew field with $l=1,2,3$.

From this representation we can deduce immediately that in particular for the quaternionic case

$$
\left\|\zeta_{M}^{\Omega_{p}}(\tau(l))\right\| \leq \frac{1}{2} \zeta_{W^{t r} W}(2)+\zeta_{W^{t r} W}(2)=\frac{3}{2} \zeta_{W^{t r} W}(2)
$$

and for the more general $(k+1)$-dimensional case

$$
\left\|\zeta_{M}^{\Omega_{p}}(\tau(i))\right\| \leq \frac{k-1}{4} \zeta_{W^{t r} W}\left(\frac{k+1}{2}\right)+\frac{k+1}{4} \zeta_{W^{t r} W}\left(\frac{k+1}{2}\right)=\frac{1}{2} k \zeta_{W^{t r} W}\left(\frac{k+1}{2}\right) .
$$

With Proposition 1 we infer that for any arbitrary index $\mathbf{n} \in \mathbb{N}_{0}^{k} \backslash\{\mathbf{0}\}$

$$
\left\|\zeta_{M}^{\Omega_{p}}(\mathbf{n})\right\| \leq \prod_{\mu=0}^{|\mathbf{n}|-1}(k+\mu) \zeta_{W^{t r} W}\left(\frac{1}{2}(k+|\mathbf{n}|)\right) .
$$

The next step is to show that we actually deal with non-trivial series. For $p \leq k$ we consider the $p$-fold periodic monogenic cotangent function associated with the lattice $\Omega_{p}$ (for details see [22] and the forthcoming paper [23]) which is defined by

$$
\cot ^{(p)}(z)= \begin{cases}q_{\mathbf{0}}(z)+\sum_{\omega \in \Omega_{k} \backslash\{0\}}\left[q_{\mathbf{0}}(z+\omega)-q_{\mathbf{0}}(\omega)\right] & \text { if } p=k \\ \sum_{\omega \in \Omega_{p}} q_{\mathbf{0}}(z+\omega) & \text { if } p<k .\end{cases}
$$

By a direct computation we obtain the Laurent expansion around the origin which reads

$$
\cot ^{(p)}(z)=q_{\mathbf{0}}(z)+2 \sum_{|\mathbf{l}|>0,|\mathbf{1}| \equiv 1(2)} V_{\mathbf{l}}(z) \zeta_{M}^{\Omega_{p}}(\mathbf{l}) .
$$

We observe that there must be some $\mathbf{l} \in \mathbb{N}_{0}^{k}$ for which $\zeta_{M}^{\Omega_{p}}(\mathbf{l}) \neq 0$, otherwise $\cot ^{(p)}-q_{\mathbf{0}}$ would be the zero function, which is a contradiction.

For $p=k+1$ we consider the monogenic $(k+1)$-fold periodic generalized $\wp$-function, introduced by A. C. Dixon in [6] for the three-dimensional case, by R. Fueter [16] for the quaternionic case, and by J. Ryan [31] for the $(k+1)$-dimensional case.

For an $i \in\{1, \ldots, k\}$ the generalized $\wp$-function associated with a $(k+1)$-dimensional lattice $\Omega_{k+1}$ denoted by $\wp_{\tau(i)}: \mathcal{A}_{k+1} \backslash \Omega_{k+1} \rightarrow \mathcal{A}_{k+1}$, is given by

$$
\wp_{\tau(i)}(z)=q_{\tau(i)}(z)+\sum_{\omega \in \Omega_{k+1} \backslash\{0\}}\left(q_{\tau(i)}(z+\omega)-q_{\tau(i)}(\omega)\right) .
$$


Its Laurent expansion around the origin turns out to be

$$
\wp_{\tau(i)}(z)=q_{\tau(i)}(z)+2 \sum_{(|\mathbf{n}|>0,|\mathbf{n}| \equiv 0(2))} V_{\mathbf{n}}(z) \zeta_{M}^{\Omega_{k+1}}(\mathbf{n}+\tau(i))
$$

and with a similar argument we conclude that there must exist some indices $\mathbf{l}$ for which $\zeta_{M}^{\Omega_{k+1}}(\mathbf{l})$ does not vanish.

This type of generalization of the Riemann zeta function to higher dimension plays a crucial role in the theory of monogenic generalizations of the classical complex analytic Eisenstein series to Clifford analysis.

\section{Generalized Eisenstein series in Clifford analysis}

One approach to arrive at the Eisenstein series $G_{n}$ in the complex plane is the Laurent expansion of Weierstraß' $\wp$ function associated with a two-dimensional lattice $\Omega=$ $\mathbb{Z} \omega_{1}+\mathbb{Z} \omega_{2}$ around the origin, reading precisely

$$
\wp(z)=\frac{1}{z^{2}}+\sum_{n=1}^{\infty}(2 n+1) G_{2 n+2}(\Omega) z^{2 n}
$$

in which the Eisenstein series

$$
G_{n}(\Omega)=\sum_{\omega \in \Omega \backslash\{0\}} \frac{1}{w^{n}} \quad(n>2, n \text { even })
$$

appear naturally. They are absolutely convergent if and only if $n>2$. Without loss of generality we can restrict ourselfes on considering the special lattice

$$
\Omega^{*}=\mathbb{Z}+\mathbb{Z} \tau \quad \text { with } \operatorname{Im}(\tau)>0
$$

since it can be transformed by a simple rotation and dilatation into a general one. Regarding the associated series $G_{n}\left(\Omega^{*}\right)$ as a function series in the upper half-plane variable $\tau$, one arrives at the classical complex analytic Eisenstein series (1).

In order to generalize the Eisenstein series $G_{n}$ to Clifford analysis we proceed in a similar way. We consider the Laurent coefficients of the generalized monogenic Weierstrassian function $\wp_{\tau(i)}$. They read $2 \zeta_{M}^{\Omega_{k+1}}(\mathbf{n}+\tau(i))$ as mentioned in the previous section. Without loss of generality we restrict ourselves on considering a special lattice

$$
\mathbb{Z} \tau+\Omega_{k}
$$

with

$$
S c(\tau)>0, \quad \text { and } \quad \Omega_{k}=\mathbb{Z} w_{1}+\ldots+\mathbb{Z} w_{k}, S c\left(w_{i}\right)=0 \quad \forall i=1, \ldots, k
$$

since any arbitrary lattice $\Omega_{k+1}$ can be transformed by a rotation into this special lattice. Then we consider the series $\zeta_{M}^{\mathbb{Z} \tau+\Omega_{k}}(\mathbf{n})$ as a function in the hypercomplex variable $\tau$ on the right half-space $H^{+}\left(\mathcal{A}_{k+1}\right)$. This motivates to introduce the following definition (cf. $[22])$ : 
Definition 3 (Generalization of the classical Eisenstein $G_{n}$ ). Let $\mathbf{n} \in \mathbb{N}_{0}^{k}$ be a multi-index with $|\mathbf{n}| \geq 2$. Then the Eisenstein series of type $G_{\mathbf{n}}: H^{+}\left(\mathcal{A}_{k+1}\right) \rightarrow \mathcal{A}_{k+1}$ are defined by

$$
G_{\mathbf{n}}(\tau)=\sum_{(\alpha, \mathbf{m}) \in \mathbb{Z} \times \Omega_{k} \backslash\{(0, \mathbf{0})\}} q_{\mathbf{n}}(\alpha \tau+\mathbf{m}) \quad(S c(\tau)>0) .
$$

We proceed to show that these series represent actually well-defined monogenic functions.

Proposition 2. The series $G_{\mathbf{n}}$ defined in (14) converge normally on the right halfspace $H^{+}\left(\mathcal{A}_{k+1}\right)$ if and only if $|\mathbf{n}| \geq 2$. Furthermore, they represent left and right monogenic functions on $H^{+}\left(\mathcal{A}_{k+1}\right)$.

Proof. As a direct consequence of Lemma 2 in combination with Proposition 1 we infer that the series $G_{\mathbf{n}}$ converge absolutely if and only if $|\mathbf{n}| \geq 2$. With any compact set $K \in H^{+}\left(\mathcal{A}_{k+1}\right)$ we can associate an arbitrary real $\varepsilon>0$ such that $K$ is strictly contained in the strip

$$
V_{\varepsilon}\left(H^{+}\left(\mathcal{A}_{k+1}\right)\right)=\left\{\tau \in H^{+}\left(\mathcal{A}_{k+1}\right):\|V e c(\tau)\| \leq \frac{1}{\varepsilon} \text { and } S c(\tau) \geq \varepsilon\right\} .
$$

These strips are subsets of $H^{+}\left(\mathcal{A}_{k+1}\right)$ with

$$
\lim _{\varepsilon \rightarrow 0} V_{\varepsilon}\left(H^{+}\left(\mathcal{A}_{k+1}\right)\right)=H^{+}\left(\mathcal{A}_{k+1}\right) .
$$

Next we show that there is a real $\rho>0$ with

$$
\|c \tau+\mathbf{d}\| \geq \rho\|c+\mathbf{d}\|
$$

for all $\tau \in V_{\varepsilon}\left(H^{+}\left(\mathcal{A}_{k+1}\right)\right)$ and $(c, \mathbf{d}) \in \mathbb{R} \times \mathbb{R}^{k}$. For $(c, \mathbf{d})=(0, \mathbf{0})$ the inequality is trivially satisfied. In the sequel we assume $(c, \mathbf{d}) \neq(0, \mathbf{0})$. We further observe that

$$
\|c \tau+\mathbf{d}\| \geq \rho\|c+\mathbf{d}\| \quad \Longleftrightarrow \quad\|\tilde{c} \tau+\tilde{\mathbf{d}}\| \geq \rho
$$

with $\tilde{c}=\frac{c}{\|c+\mathbf{d}\|}$ and $\tilde{\mathbf{d}}=\frac{\mathbf{d}}{\|c+\mathbf{d}\|}$. We observe that

$$
\tilde{c}^{2}+\|\tilde{\mathbf{d}}\|^{2}=\frac{c^{2}}{c^{2}+\|\mathbf{d}\|^{2}}+\frac{\|\mathbf{d}\|^{2}}{c^{2}+\|\mathbf{d}\|^{2}}=1 .
$$

For $\tau=x_{0}+\mathbf{x}$ we obtain

$$
\|\tilde{c} \tau+\tilde{\mathbf{d}}\|=\left\|\tilde{c} x_{0}+\tilde{c} \mathbf{x}+\tilde{\mathbf{d}}\right\|=\sqrt{\tilde{c}^{2} x_{0}^{2}+\|\tilde{c} \mathbf{x}+\tilde{\mathbf{d}}\|^{2}} \geq \sqrt{\tilde{c}^{2} \varepsilon^{2}+\|\tilde{c} \mathbf{x}+\tilde{\mathbf{d}}\|^{2}} .
$$

The set

$$
\mathcal{K}=\left\{(\mathbf{x}, \tilde{c}, \tilde{\mathbf{d}}) \in \mathbb{R}^{k} \times \mathbb{R} \times \mathbb{R}^{k}:\|\mathbf{x}\| \leq \frac{1}{\varepsilon} \text { and } \tilde{c}^{2}+\|\tilde{\mathbf{d}}\|^{2}=1\right\}
$$


is compact. The function

$$
\phi: \mathcal{K} \rightarrow \mathbb{R}^{\geq 0}, \quad(\mathbf{x}, \tilde{c}, \tilde{\mathbf{d}}) \mapsto \sqrt{\tilde{c}^{2} \varepsilon^{2}+\|\tilde{c} \mathbf{x}+\tilde{\mathbf{d}}\|^{2}}
$$

is obviously continuous on $\mathcal{K}$. Therefore, $\phi$ takes a minimum in $\mathcal{K}$.

If $c=0$, then $\phi(\mathbf{x}, \tilde{c}, \tilde{\mathbf{d}})=1>0$. If $c \neq 0$, then $\phi(\mathbf{x}, \tilde{c}, \tilde{\mathbf{d}}) \geq|\tilde{c} \varepsilon|>0$. Thus, the validity of $(15)$ is shown.

Next we apply (15) in combination with Proposition 1 under the condition that $\tau \in V_{\varepsilon}\left(H^{+}\left(\mathcal{A}_{k+1}\right)\right)$ on the expression

$$
\begin{aligned}
& \sum_{(\alpha, \mathbf{m}) \in \mathbb{Z} \times \Omega_{k} \backslash\{(0, \mathbf{0})\}}\left\|q_{\mathbf{n}}(\alpha \tau+\mathbf{m})\right\| \\
\leq & \sum_{(\alpha, \mathbf{m}) \in \mathbb{Z} \times \Omega_{k} \backslash\{(0, \mathbf{0})\}}\|\alpha \tau+\mathbf{m}\|^{-(|\mathbf{n}|+k)} \\
\leq & C \rho^{-(|\mathbf{n}|+k)} \sum_{(\alpha, \mathbf{m}) \in \mathbb{Z} \times \Omega_{k} \backslash\{(0, \mathbf{0})\}}\|\alpha+\mathbf{m}\|^{-(|\mathbf{n}|+k)} \\
& =C \rho^{-(|\mathbf{n}|+k)} \sum_{\mathbf{g} \in \mathbb{Z}^{k+1} \backslash\{0\}}\left(\mathbf{g}^{t r}\left(W^{t r} W\right) \mathbf{g}\right)^{-\frac{1}{2}(|\mathbf{n}|+k)} \\
& =C \rho^{-(|\mathbf{n}|+k)} \zeta_{W^{t r} W}\left(\frac{1}{2}(|\mathbf{n}|+k)\right) \\
< & \infty
\end{aligned}
$$

where $C$ denotes a positive real constant and $\zeta_{W^{t r} W}$ the Epstein zeta function associated with the matrix $W^{t r} W$ where $W=\left(e_{0}, w_{1}, \ldots, w_{k}\right)$. Thus, the series $G_{\mathbf{n}}$ converge normally in $H^{+}\left(\mathcal{A}_{k+1}\right)$. As a consequence of Weierstraß' convergence theorem we can infer, provided $|\mathbf{n}| \geq 2$, that the series $G_{\mathbf{n}}$ represent left and right monogenic functions in $H^{+}\left(\mathcal{A}_{k+1}\right)$, since the functions $q_{\mathbf{n}}(\alpha \tau+\mathbf{m})$ are left and right monogenic on the right half-space $H^{+}\left(\mathcal{A}_{k+1}\right)$ for $(\alpha, \mathbf{m}) \in \mathbb{Z} \times \Omega_{k} \backslash\{(0, \mathbf{0})\}$

\section{Further elementary properties :}

1. The functions $G_{\mathbf{n}}$ take all their values in $\mathcal{A}_{k+1}$.

2. If $|\mathbf{n}| \equiv 0(2)$, then the series $G_{\mathbf{n}}$ vanish identically, since the functions $q_{\mathbf{n}}$ are odd in this case.

3. If $|\mathbf{n}| \equiv 1(2)$, then the series $G_{\mathbf{n}}$ are even, because in this case the functions $q_{\mathbf{n}}$ are even. In the next section we will observe by means of the Fourier expansion that for $|\mathbf{n}| \equiv 1(2)$ these functions are really non-trivial.

4. The functions $G_{\mathbf{n}}$ have singularities of the order $(|\mathbf{n}|+k)$ precisely in the rational points with respect to the basis $\left\{w_{1}, \ldots, w_{k}\right\}$ of the dividing hyperplane

$$
T=\left\{z \in \mathcal{A}_{k+1} \mid S c(z)=0\right\}, \quad \text { i.e. in } \mathbb{Q} \omega_{1}+\ldots+\mathbb{Q} \omega_{k} .
$$


5. The series $G_{\mathbf{n}}$ are $k$-fold periodic functions with respect to the lattice $\Omega_{k}$ which follows directly by rearrangement of the series.

6. For $|\mathbf{n}| \geq 2$ the series $G_{\mathbf{n}}$ contain the monogenic Eisenstein series of type $\varepsilon_{\mathbf{n}}^{(k)}$ described in $[22,23]$ with respect to the lattice $\Omega_{k}$ which read

$$
\varepsilon_{\mathbf{n}}^{(k)}(z)=\sum_{\omega \in \Omega_{k}} q_{\mathbf{n}}(z+\omega)
$$

as a subseries or, in other words, partial derivatives of the $k$-fold periodic monogenic cotangent. The series $\varepsilon_{\mathbf{n}}^{(k)}$ generalize the complex analytic Eisenstein series of type

$$
\varepsilon_{n}(z)=\sum_{m \in \mathbb{Z}}(z+m)^{-n} \quad(n \geq 2)
$$

to Clifford analysis.

7. For any arbitrary $u \in \mathbb{Z} \backslash\{0\}$ we get

$$
\begin{aligned}
G_{\mathbf{n}}(u \tau) & =\sum_{(\alpha, \mathbf{m}) \in \mathbb{Z} \times \Omega_{k} \backslash\{(0, \mathbf{0})\}} q_{\mathbf{n}}(u \alpha \tau+\mathbf{m}) \\
& =u^{-k-|\mathbf{n}|} \sum_{(\alpha, \mathbf{m}) \in \mathbb{Z} \times \Omega_{k} \backslash\{(0, \mathbf{0})\}} q_{\mathbf{n}}\left(\alpha \tau+\frac{\mathbf{m}}{u}\right) \\
& =u^{-k-|\mathbf{n}|} G_{\mathbf{n}}\left(z ; \tilde{\Omega}_{k}\right)
\end{aligned}
$$

where $\tilde{\Omega}_{k}$ denotes the lattice $u^{-1} \Omega_{k}$ which obviously contains the original lattice $\Omega_{k}$. To obtain this formula, we have exploited the $\mathbb{R}$-homogeneity of the functions $q_{\mathbf{n}}$.

8. The set of singularities of $G_{\mathbf{n}}(\tau)$, which in the sequel will be denoted by $S$, is invariant under transformations of the form

$$
\begin{aligned}
& s \mapsto s+\mathbf{m} \quad\left(\mathbf{m} \in \Omega_{k}, s \in S\right) \\
& s \mapsto s^{-1} .
\end{aligned}
$$

The group which is generated by these types of transformations acts discontinuously on $H^{+}\left(\mathcal{A}_{k+1}\right)$ and can be regarded as a generalization of the classical modular group $S L(2, \mathbb{Z})$ for the $(k+1)$ dimensional case (compare, e.g., with [9]). 


\section{The Fourier expansion of the generalized Eisenstein series}

Since the series $G_{\mathbf{n}}$ are $k$-fold periodic and twice continuously real differentiable on the right half-space, they can be represented there by a normally convergent Fourier series of the form

$$
\sum_{\mathbf{r} \in \Omega_{k}^{\sigma}} \alpha_{f}\left(\mathbf{r}, x_{0}\right) e^{2 \pi i\langle\mathbf{r}, \mathbf{x}\rangle}
$$

where $\Omega_{k}^{\sigma}$ denotes the dual lattice of $\Omega_{k}$. Furthermore, monogenicity provides the special Fourier series representation

$$
\alpha_{f}(\mathbf{0})+\sum_{\mathbf{r} \in \Omega_{k}^{\sigma} \backslash\{\mathbf{0}\}} \alpha_{f}(\mathbf{r}) P^{+}(2 \pi \mathbf{r}, z)
$$

on the right half-space where

$$
P^{+}(\mathbf{r}, z)=\left(1+i \frac{\mathbf{r}}{\|\mathbf{r}\|}\right) e^{-\|\mathbf{r}\| x_{0}} e^{i\langle\mathbf{r}, \mathbf{x}\rangle}
$$

is the monogenic plane wave function from [5].

We will determine the Fourier expansion on $H^{+}\left(\mathcal{A}_{k+1}\right)$ of the Eisenstein series of type $G_{\mathbf{n}}(\tau)$ associated with the orthonormal lattice

$$
L_{k}=\mathbb{Z} e_{1}+\ldots+\mathbb{Z} e_{k} \subset \mathbb{R}^{k}
$$

In the sequel we assume $|\mathbf{n}| \equiv 1(2)$.

Complex function theory provides several methods to determine the Fourier expansion of the Eisenstein series of type $G_{n}$. A classical method is to determine first the Fourier expansion of the Eisenstein series $\varepsilon_{n}$ being a subseries of $G_{n}$, which admits a more direct conclusion about the Fourier series representation of $G_{n}$. We refer for example to $[11,32]$. In [32] there are also given methods how to compute the Fourier series of $G_{n}$ directly without determining the Fourier series of $\epsilon_{n}$. However, the methods applied there are still not available in Clifford analysis.

Thus, we first expand the series $\epsilon_{\mathbf{n}}^{(k)}(z)$ associated with the orthonormal lattice $L_{k}$ in $\mathbb{R}^{k}$ for $|\mathbf{n}| \geq 2$ into a Fourier series of the form

$$
\sum_{\mathbf{r} \in L_{k}} \alpha_{f}\left(\mathbf{r}, x_{0}\right) e^{2 \pi i\langle\mathbf{r}, \mathbf{x}\rangle}
$$

on the right half-space. Without loss of generality we assume $n_{1}>0$. For the sake of illustrating the integration mechanism leading to recursion formulas we will not use the multi-index notation within the following computations. For the first Fourier coefficient 
we obtain

$$
\begin{aligned}
\alpha_{f}\left(\mathbf{0}, x_{0}\right)= & \int_{0}^{1} \cdots \int_{0}^{1} \int_{0}^{1}\left(\sum_{\mathbf{m} \in L_{k}} q_{n_{1}, n_{2}, \ldots, n_{k}}(z+\mathbf{m})\right) d x_{1} d x_{2} \cdots d x_{k} \\
= & \int_{0}^{1} \cdots \int_{0}^{1} \sum_{\mathbf{m} \in L_{k}}\left(\int_{0}^{1} q_{n_{1}, n_{2}, \ldots, n_{k}}\left(x_{0}+\left[\sum_{\lambda=1}^{k} e_{\lambda} x_{\lambda}\right]+\mathbf{m}\right) d x_{1}\right) d x_{2} \cdots d x_{k} \\
= & \int_{0}^{1} \cdots \int_{0}^{1} \sum_{\mathbf{m} \in L_{k}}\left\{q_{n_{1}-1, n_{2}, \ldots, n_{k}}\left(x_{0}+e_{1}+\left[\sum_{\lambda=2}^{k} e_{\lambda} x_{\lambda}\right]+\mathbf{m}\right)\right. \\
& \left.-q_{n_{1}-1, n_{2}, \ldots, n_{k}}\left(x_{0}+\left[\sum_{\lambda=2}^{k} e_{\lambda} x_{\lambda}\right]+\mathbf{m}\right)\right\} d x_{2} \cdots d x_{k} \\
= & 0
\end{aligned}
$$

similarly to the classical complex case.

In the sequel we assume $\mathbf{r} \neq \mathbf{0}$ and compute

$$
\begin{aligned}
\alpha_{f}\left(\mathbf{r}, x_{0}\right) & =\int_{[0,1]^{k}}\left(\sum_{\mathbf{m} \in L_{k}} q_{n_{1}, n_{2}, \ldots, n_{k}}(z+\mathbf{m}) e^{-2 \pi i\langle\mathbf{r}, \mathbf{x}\rangle}\right) d x_{1} d x_{2} \cdots d x_{k} \\
& =\int_{\mathbb{R}^{k}}\left(q_{n_{1}, n_{2}, \ldots, n_{k}}(z) e^{-2 \pi i\langle\mathbf{r}, \mathbf{x}\rangle}\right) d x_{1} d x_{2} \cdots d x_{k}
\end{aligned}
$$

We first simplify this integral by applying the partial integration method successively:

$$
\begin{aligned}
\alpha_{f}\left(\mathbf{r}, x_{0}\right)= & \int_{\mathbb{R}} \cdots \int_{\mathbb{R}}\left(\int_{\mathbb{R}} q_{n_{1}, n_{2}, \cdots, n_{k}}(z) e^{-2 \pi i\langle\mathbf{r}, \mathbf{x}\rangle} d x_{1}\right) d x_{2} \cdots d x_{k} \\
= & \int_{\mathbb{R}} \cdots \int_{\mathbb{R}}\left[\left.q_{n_{1}-1, n_{2}, \ldots, n_{k}}(z) e^{-2 \pi i\langle\mathbf{r}, \mathbf{x}\rangle}\right|_{x_{1}=-\infty} ^{x_{1}=\infty}\right. \\
& \left.+2 \pi i r_{1} \int_{\mathbb{R}} q_{n_{1}-1, n_{2}, \ldots, n_{k}}(z) e^{-2 \pi i\langle\mathbf{r}, \mathbf{x}\rangle} d x_{1}\right] d x_{2} \cdots d x_{k} \\
= & (2 \pi i) r_{1} \int_{\mathbb{R}} \cdots \int_{\mathbb{R}}\left[\int_{\mathbb{R}} q_{n_{1}-1, n_{2}, \ldots, n_{k}}(z) e^{-2 \pi i\langle\mathbf{r}, \mathbf{x}\rangle} d x_{1}\right] d x_{2} \cdots d x_{k}
\end{aligned}
$$

After having applied further $n_{1}-1$ steps of partial integration with respect to $x_{1}$, we get

$$
\alpha_{f}\left(\mathbf{r}, x_{0}\right)=(2 \pi i)^{n_{1}} r_{1}^{n_{1}} \int_{\mathbb{R}^{k}} q_{0, n_{2}, \ldots, n_{k}}(z) e^{-2 \pi i\langle\mathbf{r}, \mathbf{x}\rangle} d x_{1} d x_{2} \cdots d x_{k}
$$

Now we apply the same procedure to the variables $x_{2}, \ldots, x_{k}$ which yields finally

$$
\alpha_{f}\left(\mathbf{r}, x_{0}\right)=(2 \pi i)^{|\mathbf{n}|} \mathbf{r}^{\mathbf{n}} \int_{\mathbb{R}^{k}} q_{0,0, \ldots, 0}(z) e^{-2 \pi i\langle\mathbf{r}, \mathbf{x}\rangle} d x_{1} d x_{2} \cdots d x_{k}
$$


For $x_{0}>0$ one obtains applying the residue theorem (cf. for details $[2,22]$ ) that

$$
\int_{\mathbb{R}^{k}} q_{\mathbf{0}}(z) e^{-2 \pi i\langle\mathbf{r}, \mathbf{x}\rangle} d x_{1} d x_{2} \cdots d x_{k}=\frac{A_{k+1}}{2}\left(1+i \frac{\mathbf{r}}{\|\mathbf{r}\|}\right) e^{-2 \pi\|\mathbf{r}\| x_{0}}
$$

where $A_{k+1}$ denotes the surface of the $(k+1)$-dimensional unit ball.

Finally, we get for $\varepsilon_{\mathbf{n}}^{(k)}(z)$ the following Fourier series representation on the right half-space:

$$
\varepsilon_{\mathbf{n}}^{(k)}(z)=\sum_{\mathbf{r} \in L_{k} \backslash\{\mathbf{0}\}}(2 \pi i)^{|\mathbf{n}|} \mathbf{r}^{\mathbf{n}} P_{N}^{+}(2 \pi \mathbf{r}, z)
$$

where $P_{N}^{+}$denotes the normalized monogenic exponential plane wave function which is simply said to be

$$
P_{N}^{+}(2 \pi \mathbf{r}, z)=A_{k+1} P^{+}(2 \pi \mathbf{r}, z) .
$$

Using multi-index notation, the Fourier expansion can be presented in a similar form as the Fourier expansion of the complex analytic Eisenstein series $\varepsilon_{n}$ which reads precisely (cf., e.g., [11])

$$
\varepsilon_{n}(z)=\frac{(-1)^{n}}{(n-1) !} \sum_{r \in \mathbb{N}}(2 \pi i)^{n} r^{n-1} e^{2 \pi i r z} .
$$

Furthermore, we observe that the coefficients $\alpha_{f}(\mathbf{r})$ are pure $\mathbb{C}$ scalars.

The knowledge of the Fourier expansion of the series $\varepsilon_{\mathbf{n}}^{(k)}$ will admit an explicit determination of the Fourier expansion of the series $G_{\mathbf{n}}$. We consider

$$
\begin{aligned}
G_{\mathbf{n}}(\tau) & =\sum_{\mathbf{m} \in L_{k} \backslash\{\mathbf{0}\}} q_{\mathbf{n}}(\mathbf{m})+\sum_{\alpha \in \mathbb{N}} \sum_{\mathbf{m} \in L_{k}} q_{\mathbf{n}}(\alpha \tau+\mathbf{m})+\sum_{\alpha \in \mathbb{N}} \sum_{\mathbf{m} \in L_{k}} q_{\mathbf{n}}(-\alpha \tau+\mathbf{m}) \\
& =2 \zeta_{M}^{L_{k}}(\mathbf{n})+2 \sum_{\alpha \in \mathbb{N}} \sum_{\mathbf{m} \in L_{k}} q_{\mathbf{n}}(\alpha \tau+\mathbf{m}) .
\end{aligned}
$$

Now we apply result (18) on the series $\sum_{\mathbf{m} \in L_{k}} q_{\mathbf{n}}(\alpha \tau+\mathbf{m})=\varepsilon_{\mathbf{n}}(\alpha \tau)$. Thus,

$$
\begin{aligned}
G_{\mathbf{n}}(\tau)= & 2 \zeta_{M}^{L_{k}}(\mathbf{n}) \\
& +2 \sum_{\alpha \in \mathbb{N}} \sum_{\mathbf{r} \in L_{k} \backslash\{0\}}(2 \pi i)^{|\mathbf{n}|}\left(\frac{A_{k+1}}{2}\right) \mathbf{r}^{\mathbf{n}}\left(1+i \frac{\mathbf{r}}{\|\mathbf{r}\|}\right) e^{2 \pi i\langle\mathbf{r}, \alpha \mathbf{x}\rangle} e^{-2 \pi\|\mathbf{r}\| \alpha x_{0}} \\
= & 2 \zeta_{M}^{L_{k}}(\mathbf{n}) \\
& +2(2 \pi i)^{|\mathbf{n}|}\left(\frac{A_{k+1}}{2}\right) \sum_{\alpha \in \mathbb{N}} \sum_{r \in L_{k} \backslash\{0\}} \mathbf{r}^{\mathbf{n}}\left(1+i \frac{\alpha \mathbf{r}}{\|\alpha \mathbf{r}\|}\right) e^{2 \pi i\langle\alpha \mathbf{r}, \mathbf{x}\rangle} e^{-2 \pi\|\alpha \mathbf{r}\| x_{0}} \\
= & 2 \zeta_{M}^{L_{k}}(\mathbf{n}) \\
& +2(2 \pi i)^{|\mathbf{n}|}\left(\frac{A_{k+1}}{2}\right) \sum_{\mathbf{l} \in L_{k} \backslash\{0\}}\left(\sum_{\mathbf{r} \mid \mathbf{l}} \mathbf{r}^{\mathbf{n}}\right)\left(1+i \frac{\mathbf{l}}{\|\mathbf{l}\|}\right) e^{2 \pi i\langle\mathbf{l}, \mathbf{x}\rangle} e^{-2 \pi\|\mathbf{l}\| x_{0}}
\end{aligned}
$$

where $\mathbf{r} \mid \mathbf{l}$ means that there exists an $\alpha \in \mathbb{N}$ such that $\alpha \mathbf{r}=\mathbf{l}$.

We rewrite the result in the following way: 
Theorem 2. The Fourier expansion of the series $G_{\mathbf{n}}$ on the right half-space has the representation

$$
G_{\mathbf{n}}(\tau)=2 \zeta_{M}^{L_{k}}(\mathbf{n})+2(2 \pi i)^{|\mathbf{n}|} \sum_{\mathbf{l} \in L_{k} \backslash\{0\}} \sigma_{\mathbf{n}}(\mathbf{l}) P_{N}^{+}(2 \pi \mathbf{l} ; \tau)
$$

with

$$
\sigma_{\mathbf{n}}(\mathbf{l})=\sigma_{n_{1}, \cdots, n_{k}}\left(\left(l_{1}, \ldots, l_{k}\right)\right)=\sum_{\mathbf{r} \mid \mathbf{1}} \mathbf{r}^{\mathbf{n}}
$$

\section{Remarks.}

1. By means of the Fourier series expansion, we observe that the series $G_{\mathbf{n}}$ do not vanish in the case $|\mathbf{n}| \equiv 1(2)$ which implies that we actually deal with non-trivial series.

2. We can also rewrite the multiple divisor sum in the form of an ordinary divisor sum

$$
\sigma_{\mathbf{n}}(\mathbf{l})=\sum_{\mathbf{r} \mid \mathbf{l}} \mathbf{r}^{\mathbf{n}}=\sum_{\alpha \in \mathbb{N}, \alpha \mid \operatorname{gcd}\left(l_{1}, \ldots, l_{k}\right)}\left(\frac{1}{\alpha} \mathbf{l}\right)^{\mathbf{n}}=\mathbf{l}^{\mathbf{n}} \sigma_{-|\mathbf{n}|}\left(g c d\left(l_{1}, \ldots, l_{k}\right)\right) .
$$

3. Using multi-index notation, we observe once more a similarity of the form of the Fourier expansion of $G_{\mathbf{n}}$ with the form of the Fourier expansion of the classical complex analytic Eisenstein series $G_{n}$ which reads (cf. [11])

$$
G_{n}(z)=2 \zeta(n)+\frac{2(2 \pi i)^{n}}{(n-1) !} \sum_{l \in \mathbb{N}} \sigma_{n-1}(l) e^{2 \pi i l z} .
$$

4. With respect to the generalized non-analytic Eisenstein series discussed in [8, $24,25]$ the series $G_{\mathbf{n}}$ have in common that their Fourier coefficients are composed by divisor sums in the case $\mathbf{r} \neq \mathbf{0}$ and by a variant of the Riemann zeta function in the case $\mathbf{r}=\mathbf{0}$. A significant difference concerning the structure of the Fourier expansion is that in the monogenic case the first coefficient does not depend on $x_{0}$ and that there appear monogenic exponential plane wave functions instead of Bessel functions.

The reason for the appearance of Bessel functions in the Fourier series expansion of the non-analytic Eisenstein series discussed in $[8,24,25]$ is that those non-analytic Eisenstein series are automorphic eigenfunctions of the Laplace-Beltrami operator (see [27]). Monogenicity leads to a separation of the expression $\alpha_{f}\left(\mathbf{r}, x_{0}\right)$ into an $x_{0}$ free coefficient part $\alpha_{f}(\mathbf{r})$ and a pure exponential part. 


\section{References}

[1] Brackx, F., Delanghe, R. and F. Sommen: Clifford Analysis (Pitman Research Notes: Vol. 76) Boston-London-Melbourne: Pitman 1982.

2] Cnops, J.: Introduction to Dirac Operator Theory. Lect. Notes 5th European Intensive Course on Complex Analysis and its Generalizations. Univ. Coimbra 1999.

[3] Delanghe, R.: On regular-analytic functions with values in a Clifford algebra. Math. Ann. 185 (1970), $91-111$.

[4] Delanghe, R.: On the singularities of functions with values in a Clifford algebra. Math. Ann. 196 (1972), $293-319$.

[5] Delanghe, R., Sommen, F. and V. Soucek: Clifford Algebra and Spinor Valued Functions. Dordrecht-Boston-London: Kluwer 1992.

[6] Dixon, A.: On the Newtonian potential. Quart. J. Math. 35 (1904), 283 - 296.

[7] Eisenstein, G.: Genaue Untersuchung der unendlichen Doppelproducte, aus welchen die elliptischen Functionen als Quotienten zusammengesetzt sind, und der mit ihnen zusammenhängenden Doppelreihen (als eine neue Begründung der Theorie der elliptischen Functionen, mit besonderer Berücksichtigung ihrer Analogie zu den Kreisfunctionen). Crelle's J. 35 (1847), $153-274$.

[8] Elstrodt, J., Grunewald, F. and J. Mennicke: Eisenstein series on three-dimensional hyperbolic space and imaginary quadratic number fields. J. Reine Angew. Math. 360 (1985), $160-213$.

[9] Elstrodt, J., Grunewald, F. and J. Mennicke: Vahlen's Group of Clifford matrices and spin-groups. Math. Z. 196 (1987), 369 - 390.

[10] Elstrodt, J., Grunewald, F. and J. Mennicke: Groups Acting on Hyperbolic Space. BerlinHeidelberg: Springer-Verlag 1998.

[11] Freitag, E. and R. Busam: Funktionentheorie, 2. Aufl. Berlin-Heidelberg: Springer-Verlag 1995.

[12] Fueter, R.: Über automorphe Funktionen in Bezug auf Gruppen, die in der Ebene uneigentlich diskontinuierlich sind. Crelle's J. 137 (1927), 66 - 77.

[13] Fueter, R.: Die Funktionentheorie der Differentialgleichung $\Delta u=0$ und $\Delta \Delta u=0$ mit vier reellen Variablen. Comment. Math. Helv. 7 (1934-1935), 307 - 330.

[14] Fueter, R.: Über die analytische Darstellung der regulären Funktionen einer Quaternionenvariablen. Comment. Math. Helv. 8 (1935-1936), 371 - 378.

[15] Fueter, R.: Die Singularitäten der eindeutigen regulären Funktionen einer Quaternionenvariablen, Teil I. Comment. Math. Helv. 9 (1936 - 1937), 320 - 334.

[16] Fueter, R.: Über vierfachperiodische Funktionen. Monatshefte Math. Phys. 48 (1939), $161-169$.

[17] Fueter, R.: Functions of a Hyper Complex Variable. Lect. notes written and supplemented by E. Bareiss, Fall Semester 1948/49. Zürich: Math. Inst. der Univ.

[18] Fueter, R.: Über Abelsche Funktionen von zwei Komplexen Variablen. Ann. Mat. Pura Appl. (IV Ser.) 28 (1949), $211-215$. 
[19] Iftimie, V.: Fonctions hypercomplexes. Bull. Math. Soc. Sci. Math. Rep. Soc. Roum. (Nouv. Ser. 9) 57 (1965), 279 - 332.

[20] Klingen, H.: Introductory Lectures on Siegel Modular Forms. Cambridge - New York: Cambridge University Press 1990.

[21] Koecher, M. and A. Krieg: Elliptische Funktionen und Modulformen. Berlin-Heidelberg: Springer-Verlag 1998.

[22] Kraußhar, R. S.: Eisenstein Series in Clifford Analysis (Aachener Beiträge zur Mathematik: Vol. 28; PhD Thesis). Aachen: Wissenschaftsverlag Mainz 2000.

[23] Kraußhar, R. S.: Monogenic multiperiodic functions in Clifford analysis. Complex Variables (accepted).

[24] Krieg, A.: Eisenstein series on real, complex and quaternionic half-spaces. Pac. J. Math. 133 (1988), $315-354$.

[25] Krieg, A.: Eisenstein series on the four-dimensional hyperbolic space. J. Number Theory 30 (1988), 177 - 197.

[26] Maaß, H.: Über eine neue Art von nichtanalytischen automorphen Funktionen und die Bestimmung Dirichletscher Reihen durch Funktionalgleichungen. Math. Ann. 121 (1949), $141-183$.

[27] Maaß, H.: Automorphe Funktionen von mehreren Veränderlichen und Dirichletsche Reihen. Abh. Math. Sem. Univ. Hamburg (Germany) 16 (1949), 53 - 104.

[28] Malonek, H.: Zum Holomorphiebegriff in höheren Dimensionen. Habilitation Thesis. Halle: Martin Luther Univ. Halle - Wittenberg 1987.

[29] Malonek, H.: Power series representation for monogenic functions in $\mathbb{R}^{m+1}$ based on a permutational product. Complex Variables 15 (1990), 181 - 191.

[30] Moisil, G. C. and N. Theodorescu: Fonctions holomorphes dans l'espace. Bul. Soc. Stiint. Cluij (Romania) 6 (1931), 177 - 194.

[31] Ryan, J.: Clifford analysis with generalized elliptic and quasi elliptic functions. Appl. Anal. 13 (1982), $151-171$.

[32] Terras, A.: Harmonic Analysis and Symmetric Spaces and Applications, Vol. I. New York-Berlin-Heidelberg: Springer-Verlag 1985.

[33] Weil, A.: Elliptic Functions according to Eisenstein and Kronecker. Berlin-HeidelbergNew York: Springer-Verlag 1976.

Received 02.04.2001 